\title{
Longitudinal assessment of pain management with the pain management index in cancer outpatients receiving chemotherapy
}

\author{
Akiko Fujii ${ }^{1}$ Yu Yamada $^{2}$ - Koichi Takayama ${ }^{3}$ Takako Nakano $^{1} \cdot$ Junji Kishimoto $^{4}$. \\ Tatsuya Morita ${ }^{5}$ Yoichi Nakanishi ${ }^{1}$
}

Received: 24 May 2016/Accepted: 1 November 2016/Published online: 16 November 2016

(C) The Author(s) 2016. This article is published with open access at Springerlink.com

\begin{abstract}
Purpose The adequacy of pain management for individuals with cancer who receive outpatient chemotherapy is unclear. The primary objective of this study was to assess pain prevalence and intensity in such patients. The secondary objectives included assessment of pain management with the pain management index (PMI) and exploration of predictors of inadequate pain management.

Methods Cancer patients who received outpatient chemotherapy were enrolled. Patients were required to complete questionnaires covering demographic data and including the Brief Pain Inventory and the Distress Thermometer and Impact Thermometer. The PMI score was determined twice with an interval of at least 3 weeks.

Results Of the 740 patients enrolled in the study, 524 individuals $(70.8 \%)$ completed all questionnaires. Totals of 282 patients $(53.8 \%)$ and 264 patients $(50.4 \%)$ reported pain at baseline and follow-up, respectively, with $\sim 14 \%$ of patients having moderate or severe pain at each assessment. Totals of 365
\end{abstract}

Koichi Takayama

takayama@koto.kpu-m.ac.jp

1 Research Institute for Diseases of the Chest, Graduate School of Medical Sciences, Kyushu University, Fukuoka, Japan

2 Psychiatry Division, Saitama Psychiatric Medical Center, Kitaadachi-gun, Japan

3 Department of Pulmonary Medicine, Graduate School of Medical Science, Kyoto Prefectural University of Medicine, 465 Kaiji-cho, Kawaramachi-Hirokoji, Kamigyo-ku, Kyoto 602-8566, Japan

4 Department of Research and Development of Next Generation Medicine, Kyushu University, Fukuoka, Japan

5 Department of Palliative and Supportive Care, Palliative Care Team and Seirei Hospice, Seirei Mikatahara Hospital, Hamamatsu, Japan patients $(69.7 \%)$ at baseline and 320 patients $(61.1 \%)$ at follow-up reported pain or were prescribed analgesics, with the rate of inadequate pain management for these patients being 39.7 and $51.6 \%$, respectively. Multivariable analysis for 418 patients $(79.8 \%)$ who had pain or required analgesics at baseline or follow-up (or both) revealed that the most significant predictor of inadequate pain management was depressive state.

Conclusions Pain in cancer patients receiving outpatient chemotherapy is prevalent and at risk for undertreatment. Pain management should be assessed on a regular basis and is likely to be improved by screening for depression.

Keywords Cancer · Pain · Outpatient chemotherapy · Pain management index (PMI) - Inadequate pain management . Depressive state

\section{Introduction}

Pain is one of the most frequent and burdensome symptoms in individuals with cancer [1-3]. A systematic review of 52 studies found that pain prevalence was $33 \%$ in cancer patients after curative treatment, 59\% in those undergoing anticancer therapy, and $64 \%$ in those with advanced or metastatic disease [4]. The prevalence of moderate or severe pain in patients undergoing anticancer treatment and in those with advanced, metastatic, or terminal disease was 36 and $45 \%$, respectively [4].

As a result of the recent shift in cancer care from the hospital to the outpatient setting, the number of cancer patients who receive outpatient chemotherapy has increased $[5,6]$. Given that unrelieved pain can interfere with physical function and quality of life, inadequate pain management can delay or disrupt chemotherapy [7]. The adequacy of pain management 
in cancer patients undergoing outpatient chemotherapy is unclear, however.

Many studies have applied the pain management index (PMI) to determine the adequacy of analgesic pain management in cancer patients and to explore various predictors of inadequate pain management $[2,8,9]$. A meta-analysis of 20 studies published between 2007 and 2013 found that the prevalence of inadequate pain management as determined by the PMI ranged from 4 to $68 \%$, with a weighted mean value of $31.8 \%$ [2]. Patient characteristics such as age, sex, and education have been implicated as predictors of inadequate pain management $[8,9]$. However, as far as we are aware, no previous study has found an association between the PMI and mental state such as depression, which is a common symptom in cancer patients [10]. A systematic review of 14 studies revealed that the mean prevalence of the coexistence of depression and pain in cancer patients was 36.5\% [11]. Moreover, an interaction between depression and pain is well documented in such patients [7, 12]. Depression may therefore be related to inadequate pain management.

Two cross-sectional studies and one retrospective study have previously applied the PMI to cancer patients in Japan [13-15]. To our knowledge, however, no longitudinal study with this index has been conducted in Japan. We have therefore now performed an observational longitudinal study of pain with the PMI in cancer patients undergoing outpatient chemotherapy at Kyushu University Hospital. The primary objective of our study was to assess pain prevalence and intensity in these patients. The secondary objectives were to assess the adequacy of pain management with the PMI and to explore predictors, including depressive state, of inadequate pain management during the course of outpatient chemotherapy.

\section{Patients and methods}

\section{Study design and patients}

From May 2011 to December 2013, we enrolled cancer outpatients who were newly to receive chemotherapy in the Outpatient Chemotherapy Unit (OCU) at Kyushu University Hospital in Japan. Patients were eligible for inclusion in this observational longitudinal study if they were 20 years of age or older, had been diagnosed with a solid tumor or hematologic malignancy, and had newly started outpatient chemotherapy in the OCU during the study period. Patients who had been previously treated with a chemotherapy regimen in the OCU before the onset of the present study in May 2011 and who newly visited the OCU for treatment with another regimen were also eligible. Patients were excluded if they were unable to complete questionnaires because of a visual disorder, severe mental or cognitive disorder, or inability to understand the Japanese language. Patients who were to receive preoperative chemotherapy were also excluded because their treatment schedule was not consistent with our data collection schedule.

\section{Data collection}

After they had provided informed consent, patients were required to fill in a questionnaire requesting basic information including age, sex, marital status, employment, and education. Data for the assessment of pain management were collected at baseline (when patients first received chemotherapy in the OCU) and at follow-up (at least 3 weeks later according to treatment schedule) with the use of questionnaires including the Brief Pain Inventory (BPI) and the Distress Thermometer and Impact Thermometer (DT \& IT). Given that new chemotherapy regimens are usually initiated in the inpatient setting in Japan, most patients were required to complete the pain assessments on day 1 of the second cycle and on day 1 of the third cycle of chemotherapy. However, patients who were treated with weekly or biweekly cycles of chemotherapy were required to complete the follow-up assessment at 3 or 4 weeks after the baseline assessment, respectively. Patients were excluded from the follow-up assessment if they did not visit the OCU for chemotherapy within 4 weeks after the day scheduled for the assessment. Clinical information was collected from medical records.

\section{Measures}

Pain was measured with the use of the validated Japanese version of the BPI, which asks cancer patients to describe their pain intensity at its worst, least, and average during the previous $24 \mathrm{~h}$ as well as their current pain intensity according to a Numeric Rating Scale from 0 (not present) to 10 (as bad as you can imagine) [15].

The PMI is a frequently used and well-validated tool for assessment of the adequacy of pain management for patients who have pain or taken analgesics $[1,8,13]$. It is calculated by subtraction of the patient-rated pain score from the analgesic drug score [8]. The patient-rated pain score is categorized as 0 (no pain), 1 (1-4, mild pain), 2 (5 or 6 , moderate pain), or 3 (7-10, severe pain) on the basis of the worst pain score on the BPI. The analgesic drug score is determined by the most potent analgesic drug administered: 0 (no analgesic drug), 1 (nonopioid), 2 (weak opioid), or 3 (strong opioid). The PMI can thus range from -3 (a patient with severe pain receiving no analgesic drug) to +3 (a patient receiving a strong opioid and reporting no pain). Negative PMI scores are indicative of inadequate pain management, and scores of 0 or higher are considered to reflect adequate pain management.

To explore the predictors of inadequacy of pain management, we measured depressive state using the DT \& IT, a brief 
screening tool for detection of depression in cancer patients [16]. The DT \& IT asks patients to identify the level of distress and the impact of the distress on daily life activity in the previous week using two visual analog scales with a scoring range from 0 (not present) to 10 (as bad as you can imagine) [16]. Patients who score 4 or higher on the distress scale and 3 or higher on the impact scale are considered to have a depressive state, with these cutoffs having been validated [16].

\section{Statistical analysis}

Differences in characteristics between patients included in or excluded from the analysis were evaluated with Student's $t$ test or the chi-square test. To examine predictors of inadequate pain management, we performed univariable and multivariable logistic regression analyses. Patients without pain and not taking analgesics at both assessments were excluded from logistic regression analysis because they were not considered to be at risk for pain. The outcome variable was the PMI score, which was dichotomized as 0 for a PMI of $<0$ at baseline or follow-up (or both) for inadequate pain management and as 1 for a PMI of $\geq 0$ at both assessments for adequate pain management. Independent variables included age, sex, marital status, employment, education, current chemotherapy, and depressive state. For multivariable logistic regression analysis, independent variables were selected with a stepwise procedure that was repeated until all predictors in the model met the criterion of a $P$ value of $\leq 0.10$. Statistical significance was set at a $P$ value of $<0.05$. All statistical analysis was performed with JMP 11 software (SAS Institute, Cary, NC).

\section{Results}

\section{Patient characteristics}

A total of 740 cancer patients were enrolled in the study, $524(70.8 \%)$ of whom completed all questionnaires and were included in the statistical analysis (Fig. 1). A total of 216 patients $(29.2 \%)$ was thus excluded from the analysis. The main reason for failure to complete questionnaires was cessation of current cancer treatment because of disease progression or a change of treatment plan such as transition to other chemotherapy or palliative care. Significant differences in site of primary malignancy, stage of disease, and current chemotherapy were apparent between patients included in the analysis and those excluded (Table 1).
Fig. 1 Flow diagram of patient enrollment. $a$ Number of patients who completed questionnaires including basic information. $b$ Number of patients who completed assessment at baseline or follow-up. $c$ Patient factors included distress, prolongation or cessation of chemotherapy, change in treatment plan, or death

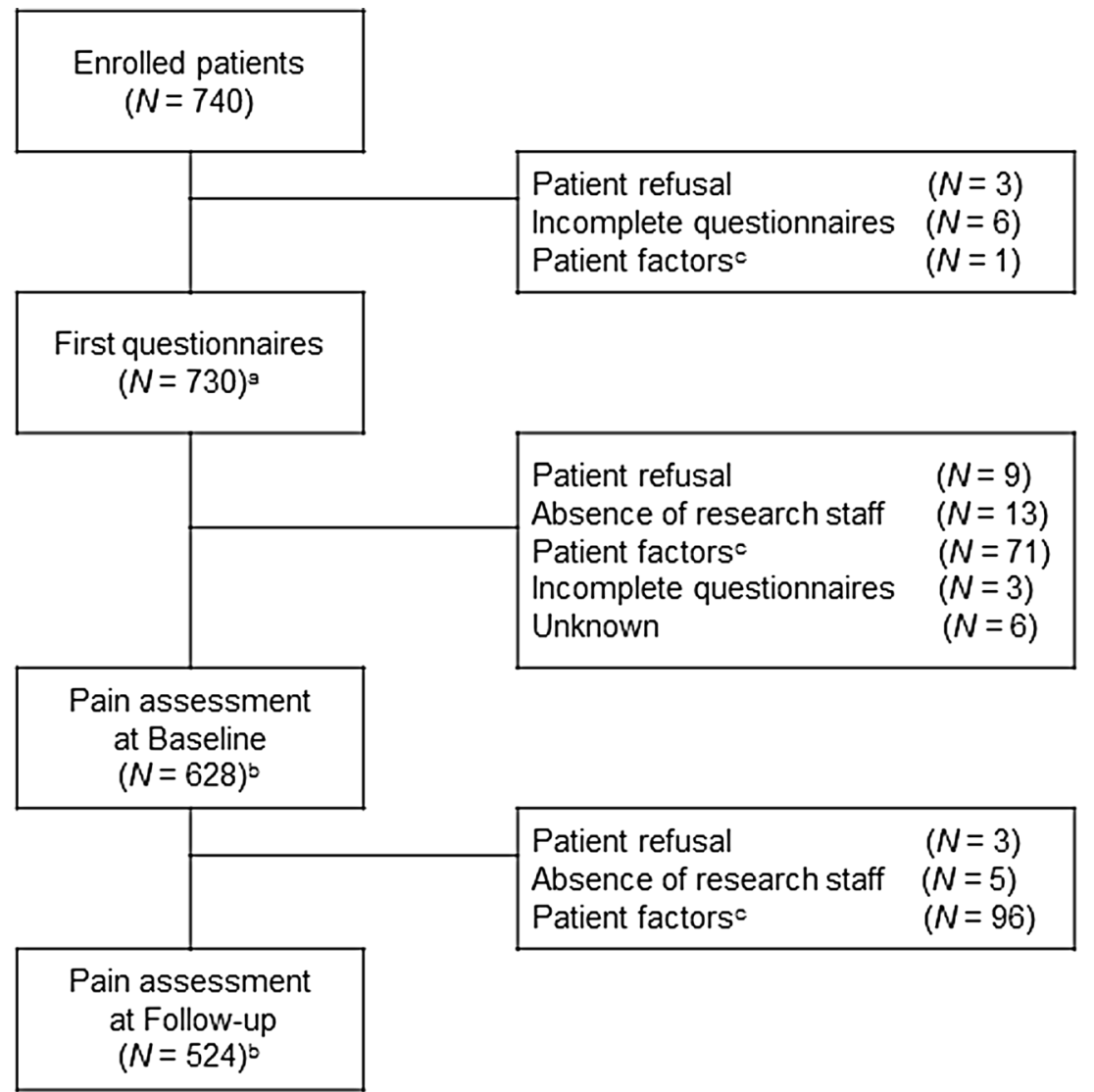


Table 1. Patient characteristics $(N=740)$

\begin{tabular}{|c|c|c|c|}
\hline Characteristic & $\begin{array}{l}\text { Patients in the analysis at } \\
\text { baseline and follow-up } \\
(N=524,70.8 \%) \\
N(\%)\end{array}$ & $\begin{array}{l}\text { Patients not in the analysis at } \\
\text { baseline and follow-up } \\
(N=216,29.2 \%) \\
N(\%)\end{array}$ & $P^{\mathrm{a}}$ \\
\hline \multicolumn{4}{|l|}{ Age (years) } \\
\hline Mean \pm SD & {$[60.8 \pm 12.1]$} & {$[62.6 \pm 10.9]$} & 0.069 \\
\hline Median (range) & {$[63(21-86)]$} & {$[63(26-84)]$} & \\
\hline Up to 63 & $279(53.2)$ & $110(50.9)$ & \\
\hline 64 or higher & $245(46.8)$ & $106(49.1)$ & 0.566 \\
\hline \multicolumn{4}{|l|}{ Sex } \\
\hline Male & $261(49.8)$ & $119(55.1)$ & \\
\hline Female & $263(50.2)$ & $97(44.9)$ & 0.191 \\
\hline \multicolumn{4}{|l|}{ Marital status } \\
\hline Married/partnered & $401(76.5)$ & $164(75.9)$ & \\
\hline Other & $123(23.5)$ & $52(24.1)$ & 0.343 \\
\hline \multicolumn{4}{|l|}{ Employment } \\
\hline Employed (full or part time) & $172(32.8)$ & $64(29.6)$ & \\
\hline Homemaker & $144(27.5)$ & 43 (19.9) & \\
\hline Not in work & $208(39.7)$ & $99(45.8)$ & \\
\hline Unknown & $0(0)$ & $10(4.6)$ & 0.063 \\
\hline \multicolumn{4}{|l|}{ Education } \\
\hline Junior high school & $67(12.8)$ & 25 (11.6) & \\
\hline High school & $268(51.1)$ & $89(41.2)$ & \\
\hline College or university & $189(36.1)$ & $89(41.2)$ & \\
\hline Unknown & $0(0)$ & $13(6.0)$ & 0.263 \\
\hline \multicolumn{4}{|l|}{ Primary cancer site } \\
\hline Lung or chest & $62(11.8)$ & $42(19.4)$ & \\
\hline Breast & $79(15.1)$ & $16(7.4)$ & \\
\hline Colon-rectum & $96(18.3)$ & $27(12.5)$ & \\
\hline Stomach or duodenum & $22(4.2)$ & $20(9.3)$ & \\
\hline Esophagus & $14(2.7)$ & $9(4.2)$ & \\
\hline Pancreas, bile duct, or liver & $130(24.8)$ & $29(13.4)$ & \\
\hline Uterus or ovary & $13(2.5)$ & $10(4.6)$ & \\
\hline Hematologic malignancy & $84(16.0)$ & $48(22.2)$ & \\
\hline Other & $24(4.6)$ & $15(6.9)$ & $<0.001$ \\
\hline \multicolumn{4}{|l|}{ UICC disease stage } \\
\hline I & $28(5.3)$ & $3(1.4)$ & \\
\hline II & $74(14.1)$ & $16(7.4)$ & \\
\hline III & $68(13.0)$ & $19(8.8)$ & \\
\hline IV & $166(31.7)$ & $87(40.3)$ & \\
\hline Recurrence & $101(19.3)$ & $41(19.0)$ & \\
\hline Unclassifiable & $84(16.0)$ & $48(22.2)$ & \\
\hline No staging or unknown & $3(0.6)$ & $2(0.9)$ & 0.002 \\
\hline \multicolumn{4}{|l|}{ Current chemotherapy } \\
\hline First line & $225(42.9)$ & $117(54.1)$ & \\
\hline Second line or higher & $124(23.7)$ & $68(31.5)$ & \\
\hline Adjuvant & $175(33.4)$ & $31(14.4)$ & $<0.001$ \\
\hline
\end{tabular}

UICC Unio Internationalis Contra Cancrum

${ }^{a} P$ values were determined with the chi-square test with the exception of that for mean age (Student's $t$ test)

Common primary sites for the malignancies of the 524 patients analyzed included colon-rectum (18.3\%), pancreas (17.7\%), breast (15.1\%), and lung (11.3\%), with $16.0 \%$ of individuals having a hematologic malignancy (Table 1). There was no significant difference in the distribution of primary cancer sites between these patients and the entire patient population treated in the OCU during the study period (data not shown). Most individuals had an Eastern Cooperative Oncology Group performance status (PS) of $0(44.6 \%)$ or 1 $(51.3 \%)$, and 175 patients $(33.4 \%)$ received adjuvant chemotherapy.

\section{Pain prevalence and analgesic prescription}

Totals of 282 patients (53.8\%) and 264 patients (50.4\%) reported pain at baseline and follow-up, respectively. Seventythree patients (13.9\%) at baseline and 74 patients (14.1\%) at follow-up reported moderate or severe pain (Fig. 2a). A total of 259 patients $(49.4 \%)$ at baseline and 181 patients (34.5\%) at follow-up had analgesics prescribed, with 48 patients $(9.2 \%)$ at baseline and 51 patients $(9.8 \%)$ at follow-up receiving weak or strong opioids (Fig. 2b). At baseline, 106 patients (20.2\%) with pain received no analgesic, with $22(4.2 \%)$ of 
Fig. 2 Pain score (a) and analgesic score (b) for 524 patients eligible for analysis at both baseline and follow-up

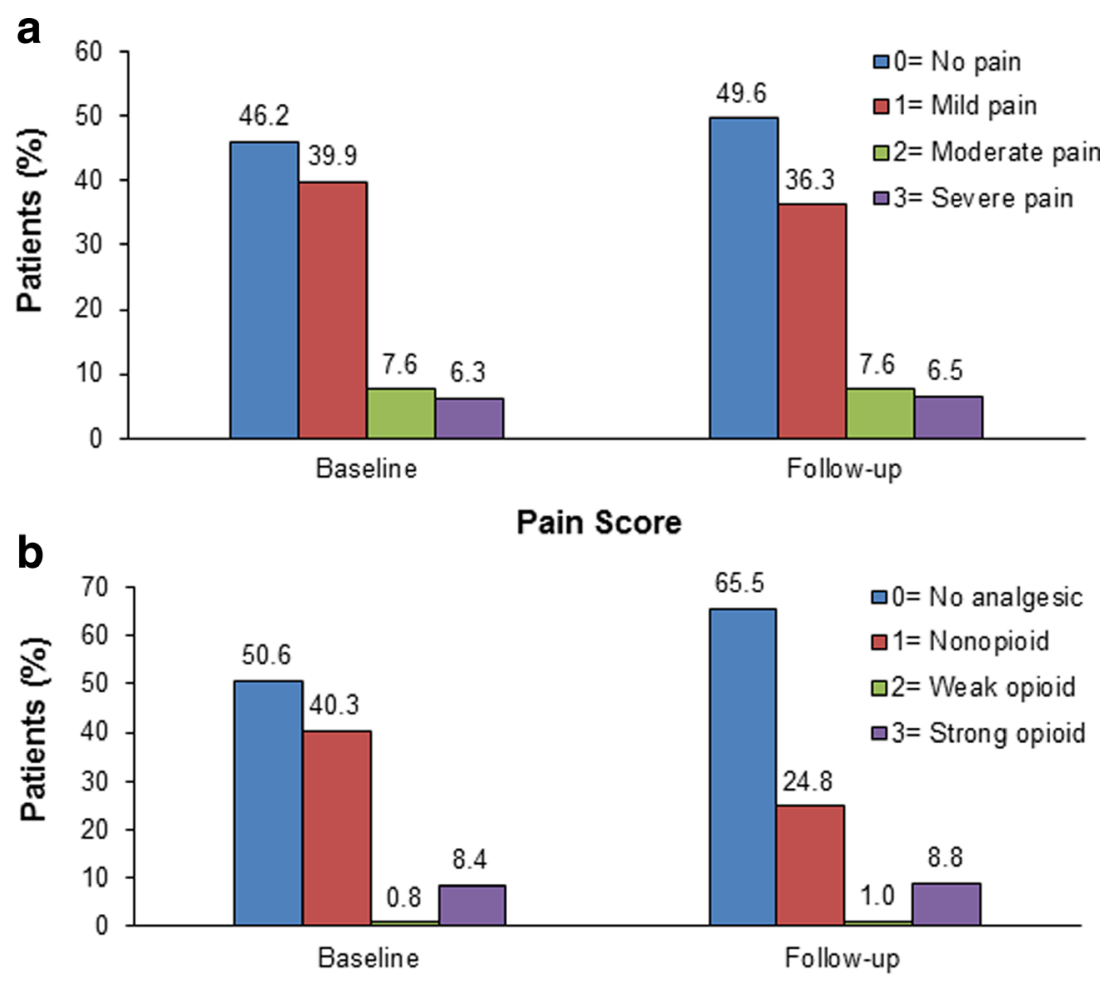

Analgesic Score these patients having moderate or severe pain and three still not being treated with any analgesic drug at follow-up. At follow-up, 139 patients (26.5\%) with pain were not treated with an analgesic drug, with $33(6.3 \%)$ of these patients reporting moderate or severe pain.

\section{Adequacy of pain management}

Totals of 365 patients $(69.7 \%)$ at baseline and 320 patients $(61.1 \%)$ at follow-up were in pain or taking analgesics (or both), with 145 of the 365 patients (39.7\%) at baseline and 165 of the 320 patients $(51.6 \%)$ at follow-up receiving inadequate pain management (Fig. 3). In addition, pain of 71 of the 145 patients $(49.0 \%)$ receiving inadequate pain management at baseline was still undertreated at follow-up.

\section{Predictors of inadequate pain management}

We excluded patients who were without pain and not taking analgesics at both assessments from the analysis of predictors of inadequate pain management. A total of 418 of the 524 patients $(79.8 \%)$ was thus included in this analysis. The results of univariable and multivariable analysis performed to identify potential predictors of inadequate pain management are
Fig. 3 Pain management index for 365 patients at baseline and 320 patients at follow-up who were in pain or taking analgesics (or both)

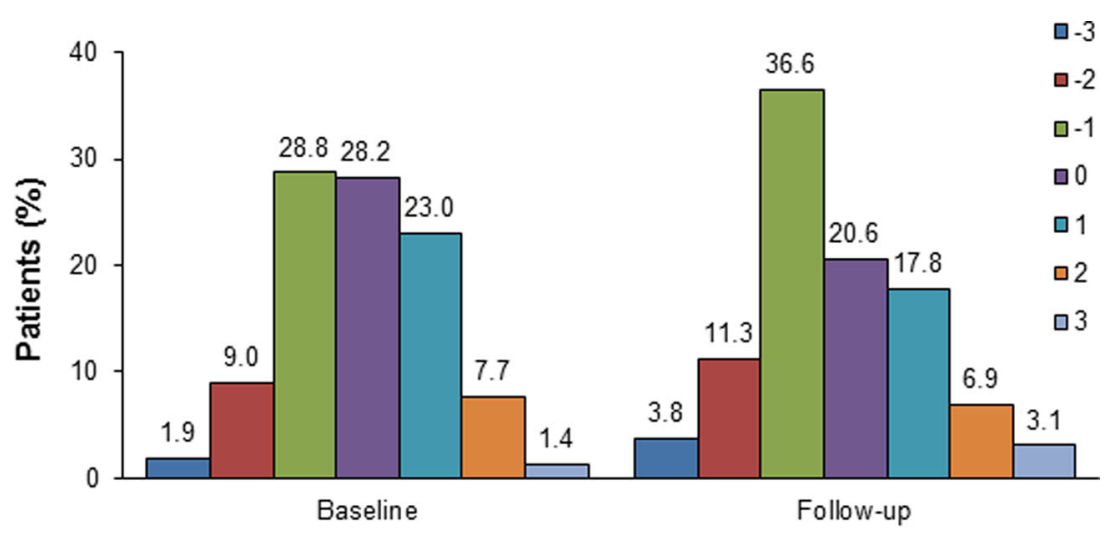

Pain Management Index 
summarized in Table 2. Multivariable analysis revealed that age, marital status, adjuvant chemotherapy, and depressive state were significantly associated with inadequate pain management. The presence of a depressive state was the strongest predictor of inadequate pain management, with an odds ratio (OR) of 2.05 (95\% confidence interval [CI] of 1.21-3.55, $P=0.006)$ in univariable analysis and an OR of $2.20(95 \%$ CI of $1.30-3.84, P=0.003$ ) in multivariable analysis.

\section{Discussion}

Our study has found that $53.8 \%$ of cancer patients experienced pain at baseline, with $13.9 \%$ reporting moderate or severe pain, and that $50.4 \%$ of patients experienced pain at followup, with $14.1 \%$ reporting moderate or severe pain. The proportion of patients with moderate or severe pain was similar to the value of $15 \%$ reported for a previous study conducted in Japan [6].

A previous analysis compared the adequacy of pain management in cancer patients between two sets of studies published between 1994 and 2007 or after 2008 and found that the mean prevalence of inadequate pain management declined from 43.4 to $31.8 \%$ [2]. Previous cross-sectional studies with small numbers of cancer patients in Japan published between 1992 and 2004 found that the rate of inadequate pain management ranged widely from 27 to $70 \%$ [13, 15]. As far as we are aware, no prospective study of the adequacy of pain management based on the PMI in cancer patients in Japan has been published since 2005 . In our study, 39.7 and $51.6 \%$ of patients with pain or taking analgesics received inadequate pain management at baseline and follow-up, respectively. The rate of inadequate pain management in our study is thus higher than that recently determined worldwide [2]. In addition, although it included patients not currently receiving cancer treatment, a previous longitudinal study of cancer outpatients found rates of inadequate pain management at baseline and after 1 month of follow-up lower than those determined in the present study [1]. Our findings therefore imply that pain management for cancer patients may not have improved in Japan in contrast to that in other countries. The facts that most patients had a good PS and that those receiving adjuvant chemotherapy were included in our study may have contributed to the relatively high

Table 2. Predictors of inadequate pain management

\begin{tabular}{|c|c|c|c|c|c|}
\hline \multirow[t]{2}{*}{ Predictors } & \multirow[t]{2}{*}{ Patients with inadequate pain management ${ }^{\mathrm{a}}$} & \multicolumn{2}{|c|}{$\begin{array}{l}\text { Univariable logistic regression } \\
(N=418)\end{array}$} & \multicolumn{2}{|c|}{$\begin{array}{l}\text { Multivariable logistic regression } \\
(N=418)\end{array}$} \\
\hline & & $P$ & OR $(95 \% \mathrm{CI})$ & $P$ & OR $(95 \% \mathrm{CI})$ \\
\hline \multicolumn{6}{|l|}{ Age (years) } \\
\hline Up to 63 & $53.3 \%(122 / 229)$ & & 1.00 & & 1.00 \\
\hline 64 or higher & $61.4 \%(116 / 189)$ & 0.10 & $1.39(0.94-2.07)$ & 0.048 & $1.49(1.00-2.24)$ \\
\hline \multicolumn{6}{|l|}{ Sex } \\
\hline Male & $54.8 \%(109 / 199)$ & & 1.00 & & \\
\hline Female & $58.9 \%(129 / 219)$ & 0.39 & $1.18(0.80-1.75)$ & & \\
\hline \multicolumn{6}{|l|}{ Marital status } \\
\hline Married/partnered & $54.4 \%(173 / 318)$ & & 1.00 & & 1.00 \\
\hline Other & $65.0 \%(65 / 100)$ & 0.06 & $1.55(0.98-2.50)$ & 0.038 & $1.64(1.03-2.66)$ \\
\hline \multicolumn{6}{|l|}{ Job } \\
\hline Other & $58.3 \%(147 / 252)$ & & 1.00 & & \\
\hline Not in work & $54.8 \%(91 / 166)$ & 0.48 & $0.87(0.58-1.29)$ & & \\
\hline \multicolumn{6}{|l|}{ Education } \\
\hline College or university & $59.1 \%(88 / 149)$ & & 1.00 & & \\
\hline Junior high school / High school & $55.8 \%(150 / 269)$ & 0.51 & $0.87(0.58-1.31)$ & & \\
\hline \multicolumn{6}{|c|}{ Current chemotherapy } \\
\hline First line or more & $54.0 \%(147 / 272)$ & & 1.00 & & 1.00 \\
\hline Adjuvant & $62.3 \%(91 / 146)$ & 0.10 & $1.41(0.93-2.13)$ & 0.048 & $1.53(1.00-2.34)$ \\
\hline \multicolumn{6}{|l|}{ Depressive state } \\
\hline No & $53.8 \%(183 / 340)$ & & 1.00 & & 1.00 \\
\hline Yes & $70.5 \%(55 / 78)$ & 0.006 & $2.05(1.21-3.55)$ & 0.003 & $2.20(1.30-3.84)$ \\
\hline
\end{tabular}

$O R$ odds ratio, $C I$ confidence interval

${ }^{a} 418$ patients who had pain or required analgesics at baseline or follow-up (or both) 
rates of inadequate pain management. Previous studies have found that a better PS and early disease stage are positive predictors of inadequate pain management, suggesting that patients in better condition tend to be considered to be in less pain $[1,9,13]$. Moreover, a previous study found that pain in patients with nonadvanced cancer currently receiving cancer therapy tended to be undertreated compared with that in patients with advanced cancer currently undergoing such therapy [1]. Indeed, we found that adjuvant chemotherapy was a significant predictor of inadequate pain management with an OR of 1.53 (95\% CI of $1.00-2.34, P=0.048$ ) in multivariable analysis.

To our knowledge, our study is the first to show an association between depressive state and inadequate pain management estimated with the PMI. Undertreatment of pain is generally attributed to an inadequate use of opioids due to barriers related to physicians, patients, family members, institutions, and society [9]. Patient-related barriers have been shown to include cognitive or affective factors such as depression and adherence to analgesic treatment $[12,17]$, consistent with our finding that a negative PMI score was associated with depressive state. Given that depression is difficult to diagnose in cancer patients because of an overlap with cancer symptoms and adverse effects of treatment, underrecognition of depression is a concern [10]. Early intervention with palliative care for physical and psychosocial symptoms in patients with metastatic non-small cell lung cancer has been found to contribute to prolongation of survival [18]. Our findings therefore highlight the importance of both physical and mental assessment in cancer patients receiving chemotherapy. If uncontrolled pain and depressive state are detected, physicians should consider consultation with a palliative care specialist in order to prevent prolongation or cessation of chemotherapy.

There are several limitations to our study. First, the enrolled patients included those with various cancer diagnoses, disease stages, and chemotherapy regimens. Our results therefore are not necessarily generalizable to specific patient populations. Second, given that the study was performed at a single institution and in a university hospital, the results may not be generalizable to outpatients receiving chemotherapy in regional hospitals. Third, we did not perform physical examinations or medical imaging to clarify the cause and mechanism of pain. Given that management of neuropathic pain is more challenging than that of nociceptive pain, the existence of neuropathic pain may have affected our results for inadequate pain management. Finally, as pointed out in previous studies $[1,9]$, the PMI does not take into account actual administration (as opposed to prescription) of analgesic drugs. Moreover, the PMI does not reflect patient satisfaction with pain management because it is calculated from pain intensity and the most potent analgesic drug prescribed [2].

In conclusion, pain is prevalent in cancer patients receiving outpatient chemotherapy and is thus a problem that warrants attention. Physicians should also be aware that depression occurs often in cancer patients and can be an obstacle to pain control. Given that symptoms may change during chemotherapy, assessment of pain management should be performed on a regular basis, and screening for depressive state should be conducted to improve such management.

Acknowledgments We thank the patients who participated in this study; S. Ikeda, K. Eto-Iwama, and S. Sakamoto for help in managing the field survey; as well as physicians (K. Kan-o, R. Ushijima, N. Noda, Y. Tsuchiya-Kawano, K. Okamura, N. Hidaka-Fujimoto, S. Anai, K. Ota, N. Kubo, and T. Hayashi of Kyushu University Hospital Cancer Center) for their assistance in enrolling patients in the study.

\section{Compliance with ethical standards}

Conflicts of interest K.T. and Y.N. have received educational grants from Janssen Pharmaceutical K.K. All remaining authors have declared no conflicts of interest.

Ethical approval The study design was approved by the institutional ethics review board of Kyushu University Hospital and complied with the 1964 Helsinki Declaration and its later amendments.

Informed consent Written informed consent was obtained from all individual participants included in the study when they visited the OCU for orientation before starting outpatient chemotherapy.

Open Access This article is distributed under the terms of the Creative Commons Attribution-NonCommercial 4.0 International License (http:// creativecommons.org/licenses/by-nc/4.0/), which permits any noncommercial use, distribution, and reproduction in any medium, provided you give appropriate credit to the original author(s) and the source, provide a link to the Creative Commons license, and indicate if changes were made.

\section{References}

1. Fisch MJ, Lee JW, Weiss M, Wagner LI, Chang VT, Cella D, Manola JB, Minasian LM, McCaskill-Stevens W, Mendoza TR, Cleeland CS (2012) Prospective, observational study of pain and analgesic prescribing in medical oncology outpatients with breast, colorectal, lung, or prostate cancer. J Clin Oncol 30:1980-1988

2. Greco MT, Roberto A, Corli O, Deandrea S, Bandieri E, Cavuto S, Apolone G (2014) Quality of cancer pain management: an update of a systematic review of undertreatment of patients with cancer. J Clin Oncol 32:4149-4154

3. von Moos R, Body JJ, Egerdie B, Stopeck A, Brown J, Fallowfield L, Patrick DL, Cleeland C, Damyanov D, Palazzo FS, Marx G, Zhou Y, Braun A, Balakumaran A, Qian Y (2016) Pain and analgesic use associated with skeletal-related events in patients with advanced cancer and bone metastases. Support Care Cancer 24:1327-1337

4. van den Beuken-van Everdingen MH, de Rijke JM, Kessels AG, Schouten HC, van Kleef M, Patijn J (2007) Prevalence of pain in patients with cancer: a systematic review of the past 40 years. Ann Oncol 18:1437-1449 
5. Dollinger M (1996) Guidelines for hospitalization for chemotherapy. Oncologist 1:107-111

6. Morita T, Fujimoto K, Namba M, Sasaki N, Ito T, Yamada C, Ohba A, Hiroyoshi M, Niwa H, Yamada T, Noda T (2008) Palliative care needs of cancer outpatients receiving chemotherapy: an audit of a clinical screening project. Support Care Cancer 16:101-107

7. Wang HL, Kroenke K, Wu J, Tu W, Theobald D, Rawl SM (2012) Predictors of cancer-related pain improvement over time. Psychosom Med 74:642-647

8. Cleeland CS, Gonin R, Hatfield AK, Edmonson JH, Blum RH, Stewart JA, Pandya KJ (1994) Pain and its treatment in outpatients with metastatic cancer. N Engl J Med 330:592-596

9. Deandrea S, Montanari M, Moja L, Apolone G (2008) Prevalence of undertreatment in cancer pain. A review of published literature. Ann Oncol 19:1985-1991

10. Nelson CJ, Cho C, Berk AR, Holland J, Roth AJ (2010) Are gold standard depression measures appropriate for use in geriatric cancer patients? A systematic evaluation of self-report depression instruments used with geriatric, cancer, and geriatric cancer samples. J Clin Oncol 28:348-356

11. Laird BJ, Boyd AC, Colvin LA, Fallon MT (2009) Are cancer pain and depression interdependent? A systematic review. PsychoOncology 18:459-464

12. Kwon JH, Oh SY, Chisholm G, Lee JA, Lee JJ, Park KW, Nam SH, Song HH, Lee K, Zang DY, Kim HY, Choi DR, Kim HJ, Kim JH,
Jung JY, Jang G, Kim HS, Won JY, Bruera E (2013) Predictors of high score patient-reported barriers to controlling cancer pain: a preliminary report. Support Care Cancer 21:1175-1183

13. Okuyama T, Wang XS, Akechi T, Mendoza TR, Hosaka T, Cleeland CS, Uchitomi Y (2004) Adequacy of cancer pain management in a Japanese cancer hospital. Jpn J Clin Oncol 34:37-42

14. Tateno Y, Ishikawa S (2012) Clinical pathways can improve the quality of pain management in home palliative care in remote locations: retrospective study on Kozu Island. Japan Rural Remote Health 12:1992

15. Uki J, Mendoza T, Cleeland CS, Nakamura Y, Takeda F (1998) A brief cancer pain assessment tool in Japanese: the utility of the Japanese brief pain inventory-BPI-J. J Pain Symptom Management 16:364-373

16. Akizuki N, Yamawaki S, Akechi T, Nakano T, Uchitomi Y (2005) Development of an impact thermometer for use in combination with the distress thermometer as a brief screening tool for adjustment disorders and/or major depression in cancer patients. J Pain Symptom Management 29:91-99

17. Kwon JH (2014) Overcoming barriers in cancer pain management. $\mathrm{J}$ Clin Oncol 32:1727-1733

18. Temel JS, Greer JA, Muzikansky A, Gallagher ER, Admane S, Jackson VA, Dahlin CM, Blinderman CD, Jacobsen J, Pirl WF, Billings JA, Lynch TJ (2010) Early palliative care for patients with metastatic non-small-cell lung cancer. N Engl J Med 363:733-742 